\title{
Linking the gut microbiota to human health
}

\author{
Virginia Robles Alonso* and Francisco Guarner \\ Digestive System Research Unit, University Hospital Vall d'Hebron, Ciberehd, Passeig Vall d'Hebron 119-129, \\ 08035 Barcelona, Spain
}

\section{Abstract}

The human gut is the natural environment for a diverse and dynamic microbial ecosystem, whose structure and functions are presently a major target of research in biomedicine. Experimental studies in germ-free animals performed some decades ago revealed the importance of these microbial communities for normal growth and development and for the maintenance of health in adult life. The host provides habitat and nutrition to the microbial communities and derives many benefits from its symbionts that contribute to metabolic, defensive and trophic functions. Development of novel gene sequencing technologies as well as availability of powerful bioinformatic analysis tools provide new insights into the composition and structure of the human gut microbiota. There is no clear definition of the characteristics of a normal 'healthy' gut microbiota in human subjects, but several disease states have been associated with changes in the composition of faecal and intestinal mucosal communities, including inflammatory bowel diseases, obesity and the metabolic syndrome. Probiotics and prebiotics are used to improve symbiosis between enteric microbiota and the host or restore states of dysbiosis.

\section{Key words: Enterotypes: Metagenomics: Microbiome: Probiotics: Prebiotics: Symbiosis}

\section{Host-microbe relationships in the gut}

Until recent years, our knowledge on the human gut microbiota was largely limited to community members with potential pathogenicity. Certain bacteria of the human gut microbiota are able to invade the mucosal barrier or translocate into internal body compartments causing local and systemic infections. Some other bacteria are associated with toxin formation and induce disease when they become dominant. Most of these potential pathogens were isolated in culture and recognised by traditional diagnostic techniques. However, the large majority of bacteria in the human gut were not grown in standard culture media and their potential role in health or disease has been ignored. The culturability of certain phylotypes seems to be related to their relative abundance ${ }^{(1)}$.

The concept of a host-microbe symbiotic relationship in the gut, in terms of proven benefits or mutualism among partners, is mostly based on studies carried out with germfree animals ${ }^{(2)}$. Such studies have demonstrated important differences between germ-free and colonised animals in body anatomy and physiology (Fig. 1), and interestingly, reconstitution of germ-free animals with a microbiota by faecal implants from conventional counterparts restores most of the deficiencies ${ }^{(3)}$. These experiments indicated that microbial colonisation has important and specific impacts on body growth and development as well as on the maintenance of health during adult life. The main functions of the gut microbiota are ascribed into three categories, i.e. metabolic, protective and trophic functions ${ }^{(4)}$.

Microbial communities of symbionts are composed of different cell lineages and provide the host with a huge diversity of genes and metabolic functions. In this way, symbionts can enhance the ability of the host to acquire nutrients from the environment or provide the pathways for synthesis of essential organic compounds ${ }^{(5)}$. The vast majority of bacteria in the colon are strict anaerobes and thus derive energy from the saccharolytic fermentation of non-digestible substrates, such as complex plant carbohydrates (dietary fibre), providing energy and nutrients for both host and symbiont communities. Carbohydrates in the colon are fermented to SCFA: acetate, propionate and butyrate. These compounds are all used to some degree by mucosal epithelial cells to obtain energy. Butyrate is of particular interest, as it may also modulate epithelial cell growth and differentiation. Epithelial cells in the distal colon derive $60-70 \%$ of their energy requirements from bacterial fermentation products ${ }^{(6)}$. SCFA also contribute to host homeostasis by acidifying the luminal $\mathrm{pH}$ and thus inhibiting the growth of pathogens. In fact, the so-called clinical entity 'diversion colitis', which occurs in diverted segments of the large bowel excluded from faecal transit, improves after treatment with a local perfusion of SCFA ${ }^{(7)}$. Undigested dietary proteins reaching the colon and proteins from desquamed epithelial cells are fermented to branched-chain fatty acids

Abbreviation: IBD, inflammatory bowel disease.

*Corresponding author: Dra. V. Robles Alonso, email vrobles@vhebron.net 
Impact of the microbiota on host anatomy and physiology

Germ-free v. colonised mice

Nutrition, growth and development
Reduced:
Organ weight (heart, liver, lungs)
Cardiac output
Oxygen consumption
Increased:
Food intake

Immune system
Reduced:
Mesenteric and systemic lymph nodes
Mucosa-associated Iymphoid tissue
Serum Ig levels
Increased:
Susceptibility to infection

Fig. 1. Body size and organ weights (heart, lung and liver), as well as cardiac output and oxygen consumption, are significantly reduced in germ-free mice when compared with conventionally colonised counterparts. However, the intake of food is higher in germ-free mice. This observation suggests that microbial colonisation plays a role in body growth and development, as well as in energy harvesting from food. On the other hand, mucosal lymphoid tissues, lymph nodes and serum $\gamma$-globulin levels are reduced in germ-free animals, whereas susceptibility to infection is highly increased. This indicates that colonisation is needed for a normal development of the immune system.

like isobutyrate, isovalerate and some nitrogenous and sulphur-containing compounds, some of which may be toxic to the host. Consequently, excessive fermentation of proteins in the distal colon is suspected to play a detrimental role in certain disease states such as colon cancer and inflammatory bowel disease $(\mathrm{IBD})^{(4)}$

Immune tissues in the gastrointestinal tract constitute the largest and most complex fraction of the human immune system. Intestinal epithelial cells lie in close contact with luminal contents, and are key players in signalling and mediating host innate and adaptive mucosal immune responses ${ }^{(8)}$. It is estimated that at least $80 \%$ of the antibody production of the body takes place locally in the gut lamina propria, mainly providing dimeric $\operatorname{Ig} \mathrm{A}^{(9)}$. The intestinal mucosa is a large surface exposed to environmental pathogenic and non-pathogenic antigens, and the gut microbes play a critical role in the development of a robust and balanced immune system. Innocuous soluble antigens and commensal microbes stimulate secretory immune responses and induce regulatory pathways for the suppression of pro-inflammatory Th2dependent responses (including IgE antibodies), Th1dependent delayed-type hypersensitivity, IgG antibodies and Th17-dependent granulocytic reactions. Immune homeostasis results from complex mechanisms in the gut, including the active phenomenon of 'oral tolerance', where mucosal regulatory $\mathrm{T}$ cells are major players. Interestingly, germ-free mice are immunodeficient and highly susceptible to pathogen or opportunistic infections ${ }^{(10-13)}$. These mice show low densities of lymphoid cells in the gut mucosa and low levels of serum Ig. At the same time, they fail to develop normal adaptation to dietary antigens like ovo-albumin, and oral tolerance mechanisms are depressed or abrogated. Besides the homeostatic regulation of the immune system, other trophic functions of the microbiota include the control of epithelial cell proliferation and differentiation and modulation of certain neuroendocrine pathways.

\section{The human gut microbiota}

Recent developments in molecular biology have allowed more accurate investigation of microbial communities in environmental samples, as the new techniques are culture independent $^{(14)}$. As mentioned, a large proportion of the bacteria living in the human gut cannot be isolated and characterised by traditional culture methods ${ }^{(4)}$. Molecular biological techniques are based on the differences in the sequence of nucleotides of the microbial genes. The majority of these techniques consist of the extraction of DNA from the sample, followed by amplification and sequencing of $16 \mathrm{~S}$ ribosomal RNA genes, which contain conserved and variable regions that allow taxonomic identification ranging from the domain and phylum level to the species level. Currently, about two million aligned and annotated 16S rRNA sequences are available in DNA databases (http://rdp.cme.msu.edu/). Taxonomic identification is based on sequence similarity analysis of $16 \mathrm{~S}$ rRNA, comparing samples with reference sequences in the database. In this way, studies on the 16S rRNA gene provide information about microbial composition and diversity of species in a given sample. The most recent development in the analysis of the gut microbiota is the so-called 'metagenomics', described as the study of all the genetic material recovered directly from environmental samples, bypassing the need to isolate and culture individual bacterial community members ${ }^{(15)}$. The metagenome is the collective genetic content of the combined genomes of the constituents of an ecological community. The microbiome is defined as the collective genome of the microbial symbionts in a host animal ${ }^{(16)}$. The metagenomic approach has the advantage of not only providing the phylogenetical characterisation of the microbial community, but also can be telling about biological functions present in the community.

The large intestine is the principal site of permanent microbial colonisation in the human body. Estimates suggest that the colon harbours over $10^{14}$ microbial cells, i.e. several hundred grams of microbes, most of them belonging to the domain Bacteria. Molecular studies based on 16S rRNA gene sequencing have highlighted that only seven to nine of the fifty-five known divisions or phyla of the domain Bacteria are detected in faecal or mucosal samples from the human gut $^{(15-18)}$. Moreover, such studies also revealed that more than $90 \%$ of all the phylotypes (sequences with $97 \%$ identity that represent a single species) belong to just two divisions: the Bacteroidetes and the Firmicutes (Fig. 2). The other divisions that have been consistently found in samples from the human distal gut are Proteobacteria, Actinobacteria, Fusobacteria and Verrucomicrobia. Of the thirteen divisions of the domain Archea, only one or two species seem to be represented in the human distal gut microbiota. Thus, at the division level, the human intestinal ecosystem is less diverse than other ecosystems on Earth, like soils and ocean waters, which may contain twenty or more divisions ${ }^{(17)}$. However, at 


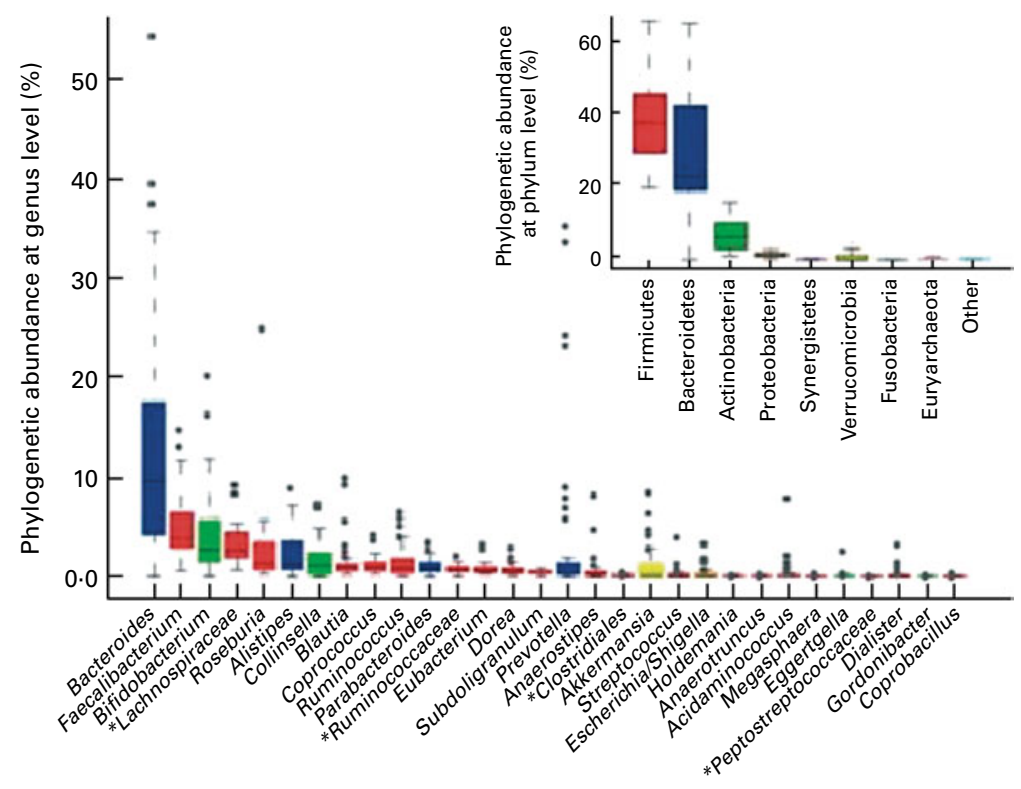

Fig. 2. Genus abundance variation box plot for the thirty most abundant genera of the human gut microbiota, as determined by metagenomic sequencing of human faecal samples. Genera are coloured by their respective phylum (see inset for colour key). Inset shows phylum abundance box plot. Genus- and phylum-level abundances were measured using reference-genome-based mapping (source: from Fig. 1(b) in: Arumugam M, Raes J, Pelletier E, et al. (2011) Enterotypes of the human gut microbiome. Nature 473, 174-180; with permission).

lower taxonomic levels (species or strain), there is considerable variation in the composition of the faecal microbiota among human individuals. Strain diversity between individuals is highly variable so that studies have found that a large proportion of the identified strain-level phylotypes are unique to each person $^{(17)}$. Each individual harbours his or her own distinctive pattern of bacterial composition.

In a cohort of 124 European adult subjects, a total of $3 \cdot 3$ million microbial genes were characterised by metagenomic analysis of faecal samples ${ }^{(18)}$. This effort has provided the first gene catalogue of the human gut microbiome. Each individual carries an average of 600000 non-redundant microbial genes in the gastrointestinal tract. This figure suggests that most of the 3.3 million genes in the catalogue are shared. It was found that about 300000 microbial genes are common, in that they are present in at least $50 \%$ of individuals. Up to $98 \%$ of genes in the catalogue are bacterial, and that the entire cohort of individuals harbours between 1000 and 1150 prevalent bacterial species, with at least 160 species per individual ${ }^{(18)}$. Interestingly, Bacteroides, Faecalibacterium and Bifidobacterium are the most abundant genera, but their relative abundance is highly variable across individuals.

Network analysis of species abundance across different individuals suggested that the overall structure of the human gut microbiota in each individual conforms to discrete and distinct patterns defined by interactions within community members ${ }^{(19)}$. This hypothesis was investigated using a dataset of metagenomic sequences from American, European and Japanese individuals. The phylogenteic analysis for taxonomic assignments was performed by mapping the metagenomic sequences to the reference genomes of fully sequenced bacteria. Multidimensional cluster analysis and principal component analysis revealed that all individual samples formed three robust clusters that have been designated as 'enterotypes ${ }^{(19)}$. Each of the three enterotypes is identifiable by the variation in the levels of one of three genera: Bacteroides (enterotype 1), Prevotella (enterotype 2) and Ruminococcus (enterotype 3). The enterotype concept suggests that enteric microbiota variations across individuals are generally stratified, not continuous. This further indicates the existence of a limited number of well-balanced hostmicrobial symbiotic states. However, other investigators have recently shown that there was little or no segregation into stool enterotypes when samples are analysed at strain-level phylotype using the $16 \mathrm{~S}$ rRNA sequencing approach ${ }^{(20)}$. This further reinforces the notion of high variability in the human gut microbiome when defined at a strain level.

The full potential of the molecular approach includes metatranscriptomics, metaproteomics and metabolomics, which analyse RNA, proteins and metabolites, respectively, of complex communities. Environmental metatranscriptomics retrieves and sequences environmental mRNA from a microbial ecosystem to assess what genes may be expressed in that community. Metaproteomics allows us to link the abundance and activity of enzymes to their phylogenetic origin based on proteins. Lastly, the metabolome is the terminal downstream product of the genome and consists of the total complement of all the low-molecular-weight molecules in a cell, tissue or organism. Based on this, the concept of 'global metabolic profiling', which provides a unique overview of the metabolic state of an individual, has led to exploration of the effect of the gut microbiota on drug metabolism and toxicity. 


\section{The concept of dysbiosis}

An imbalance of the normal gut microbiota composition is called dysbiosis. A number of disease states have been associated with changes in the composition of the gut microbiota. For instance, antibiotic-associated diarrhoea is caused by an altered balance in gut microbial communities, resulting in both decreased fermentation of indigestible carbohydrates and rapid overgrowth of opportunistic micro-organisms with potential pathogenicity. It ranges from mild episodes that resolve when antibiotics are stopped to serious complications such as toxic megacolon, bowel perforation and death. Risk is increased with different clinical conditions such as extremes of age, co-morbidity, oral broad spectrum antibiotics, prolonged antibiotic duration, previous episodes of antibiotic-associated diarrhoea and hospitalisation.

Intestinal bacteria play a role in the pathogenesis of sepsis by bacterial translocation, defined as the passage of viable bacteria from the gastrointestinal tract through the epithelial mucosa. Extensive work on bacterial translocation has been performed in animal models and occurs notably in haemorrhagic shock, burn injury, trauma, intestinal ischaemia, intestinal obstruction, severe pancreatitis, acute liver failure and cirrhosis ${ }^{(21)}$.

Some recent data on the metabolic syndrome suggest that changes in gut microbiome composition may play a role in the disorder. Studies performed in mice reveal a shift in the abundance of Bacteroidetes and Firmicutes ${ }^{(22)}$. Also, in animal models, it seems that transplantation of gut microbiota from obese mice to non-obese, germ-free mice resulted in transfer of metabolic syndrome-associated features from the donor to the recipient ${ }^{(23)}$. The mechanisms advocated are the provision of additional energy by the conversion of dietary fibre to SCFA, effects on gut-hormone production and increased intestinal permeability, causing elevated systemic levels of lipopolysaccharides. The contact with these antigens seems to contribute to low-grade inflammation, a characteristic trait of obesity and the metabolic syndrome. Presumably, obesity affects the diversity of the gut microbiota and, probably, the way individuals harvest energy from nutrients. It needs to be highlighted that between obese and lean individuals there may be dramatic differences in diet, so that we cannot rule out whether diet or other undetermined variables may also account for the observed microbiome changes. Further research on human subjects must be conducted.

One of the major hypotheses underlying the pathogenesis of IBD is the presence of abnormal communication between gut microbial communities and the mucosal immune system $^{(24)}$. Luminal bacteria appear to provide the stimulus for immunoinflammatory responses, leading to mucosal injury. The normal mucosal defence relies mainly on the production of IgA antibodies that are secreted into the gut lumen where they neutralise microbes, thus avoiding mucosal inflammation. In IBD, however, mucosal production of IgG antibodies against intestinal bacteria is highly increased. Besides, mucosal T-lymphocytes are hyper-reactive against antigens of the common intestinal microbiota. The altered immune response is not specifically addressed towards a single group of potential pathogens, but involves a large and undefined number of commensal species belonging to the common enteric flora. There is also some evidence showing that the microbiota of patients with IBD differs from that of healthy subjects. Differences include low biodiversity of dominant bacteria, high variability over time and changes both in composition and spatial distribution (high concentrations of mucosal adherent bacteria). The microbiota of Crohn's disease patients is characterised by a decreased diversity within the Clostridium leptum group, with one species, Faecalibacterium prausnitzii, described as anti-inflammatory ${ }^{(25)}$. In addition, increased numbers of the Proteobacteria and Actinobacteria phyla have also been observed ${ }^{(26)}$.

Autoimmunity and related disorders such as atopies have been also associated with a dysfunction of gut microbial colonisation. A number of studies using molecular techniques confirm that the gut microbial ecosystem differs between children with and without atopic eczema. One hypothetical underlying mechanism is the mode and place of delivery. In fact, children born by means of caesarean section are colonised by bacteria originating from the hospital environment and not from maternal skin ${ }^{(27)}$. A cohort study by Penders et al. ${ }^{(28)}$ suggests that early colonisation by Escherichia coli increases the risk of developing eczema and colonisation with Clostridium difficile is associated with a higher risk of eczema, recurrent wheeze and allergic sensitisation. Undoubtedly, there are other environmental conditions, such as feeding regimen, antibiotic intake or hygiene, which are well-recognised risk factors.

Bacterial colonisation of the intestine plays a major role in the post-natal development and maturation of the immune and endocrine systems and, therefore, the central nervous system. Experimental data suggest a role of enteric bacteria in the gut-brain axis. Irritable bowel syndrome often develops after an acute infectious diarrhoea episode. In this context, there is a substantial body of evidence to link the pathogenesis of irritable bowel syndrome with gut bacteria. A dysbiosis of different bacterial groups has been detected, some of them involved in bile acid transformation ${ }^{(29)}$. Autism is a disorder of neural development characterised by impaired social interaction and communication, and by restricted and repetitive behaviour. Symptoms typically become apparent before a child is 3 years old. Some evidence supports the alterations of the faecal microbiota in patients with autism, with an increase in several subtypes of Clostridium ${ }^{(30,31)}$. In some cases, treatment with non-absorbed antibiotics can improve symptoms.

\section{Therapeutical approaches to dysbiosis}

Even if associations of dysbiosis with disease do not necessarily predict cause-effect relationships (microbial changes might be consequence of disease rather than a primary event), there is growing interest in developing strategies to improve the 'physiological' quality of the human gut microbial ecosystem for health benefits. Different interventional approaches have emerged, including the use of antibiotics, probiotics, prebiotics, combinations of probiotics and prebiotics, or techniques for microbial reconstitution though faecal transplantation. The referred approaches aim at improving 
host-microbe symbiosis in the gut by combating overgrowth of opportunistic community members or providing live micro-organism or metabolic substrates in order to promote growth and activity of beneficial species.

Probiotics were defined as live micro-organisms which, when administered in adequate amounts as part of food, confer a health benefit on the host', as proposed by the Joint FAO/WHO Expert Consultation in 2001. Use of probiotics has shown benefit in the prevention or treatment of some disorders, including lactose malabsorption, acute diarrhoea, irritable bowel syndrome, necrotising enterocolitis, mild forms of IBD and others. A guideline for the use of probiotics and prebiotics in gastroenterology was recently updated by the World Gastroenterology Organization ${ }^{(32)}$

The term prebiotic refers to 'a selectively fermented ingredient that allows specific changes, both in the composition and/or activity in the gastrointestinal microbiota that confers benefits upon host well-being and health'. Concurrently, a prebiotic should not be hydrolysed by human intestinal enzymes; it should be selectively fermented by beneficial bacteria and this selective fermentation should result in beneficial effects on health or well-being of the host ${ }^{(33)}$. The combination of probiotics and prebiotics is termed 'synbiotic', and is an exciting concept aimed at optimising the impact of probiotics on the gut microbial ecosystem. A large number of human intervention studies have been performed that have demonstrated that dietary consumption of certain food products can result in statistically significant changes in the composition of the gut microbiota in line with the prebiotic concept ${ }^{(33)}$.

Up to date, prebiotic effects have been demonstrated by the consumption of inulin-type fructans or galactooligosaccharides. The former ones are naturally available in a wide variety of food products such as wheat, barley, onion, garlic, banana and tomato. Galacto-oligosaccharides are available as commercially products derived from lactose, but some of them are naturally present in human milk. The characteristic composition of human milk is associated with a bifidogenic effect in breast-fed neonates ${ }^{(34)}$. Human milk is rich in oligosaccharides that are resistant to digestive processes and reach the colon. Cow and human milk have significant qualitative and quantitative differences regarding these carbohydrates, and the bifidogenic effect of human milk is not observed in infants fed with cows' milk-based formulas. Because of their peculiar chemical structure, human milk oligosaccharides have a very significant role in modulating the intestinal microbiota of neonates.

In addition, there is evidence from controlled trials ${ }^{(35)}$ showing that prebiotics are able to reduce the incidence of atopic diseases with a sustained effect over the time. Besides, a reduction in the risk of some infectious diseases, such as respiratory tract infections, is likely. These data need to be confirmed for effectiveness. It is important to highlight that the available data on prebiotic effects do not demonstrate adverse effects.

Finally, faecal transplant has emerged as an alternative approach to treat relapsing diarrhoea by $C$. difficile infection. This procedure has shown success in a subset of patients who failed to standard treatment, with reported response rates up to $87 \%{ }^{(36)}$. A total of 239 patients who had undergone faecal transplantation were reported. Seventeen of twentytwo studies of faecal transplantation were performed in patients with fulminant or refractory $C$. difficile infection. The major concern about this approach is the potential risk of transmission of infectious diseases ${ }^{(36)}$.

In summary, the development of novel gene sequencing technologies as well as the availability of powerful bioinformatic analysis tools have allowed a dramatic proliferation of research work on the human gut microbiota. Large-scale studies are providing a deeper insight on the microbial communities that usually inhabit the human gut, and allow the identification of changes that are associated with disease states, helping us to combat them.

\section{Acknowledgements}

The contribution made by each author was as follows: V. R. A. wrote the manuscript and F. G. reviewed the draft. There are no conflicts of interest. Ciberehd is funded by the Instituto de Salud Carlos III.

\section{References}

1. Walker AW, Ince J \& Duncan SH (2011) Dominant and dietresponsive groups of bacteria within the human colonic microbiota. ISME J 5, 220-230.

2. Wostmann BS (1981) The germfree animal in nutritional studies. Annu Rev Nutr 1, 257-279.

3. O'Hara AM \& Shanahan F (2006) The gut flora as a forgotten organ. EMBO Rep 7, 688-693.

4. Guarner F \& Malagelada JR (2003) Gut flora in health and disease. Lancet 361, 512-519.

5. Moran NA (2006) Symbiosis. Curr Biol 16, R866-R871.

6. Roediger WE (1989) Short chain fatty acids as metabolic regulators of ion absorption in the colon. Acta Vet Scand Suppl 86, 116-125.

7. Kiely EM, Ajayi NA, Wheeler RA, et al. (2001) Diversion procto-colitis: response to treatment with short-chain fatty acids. J Pediatr Surg 36, 1514-1517.

8. Kagnoff MF (2006) Microbial-epithelial cell crosstalk during inflammation: the host response. Ann N Y Acad Sci 1072, 313-320.

9. Brandtzaeg P (2009) Mucosal immunity: induction, dissemination, and effector functions. Scand J Immunol $\mathbf{7 0}$, $505-515$

10. Cebra JJ (1999) Influences of microbiota on intestinal immune system development. Am J Clin Nutr $\mathbf{6 9}$, 1046S-1051S.

11. Umesaki Y, Setoyama H, Matsumoto S, et al. (1999) Differential roles of segmented filamentous bacteria and clostridia in development of the intestinal immune system. Infect Immun 67, 3504-3511.

12. Umesaki Y \& Setoyama H (2000) Structure of the intestinal flora responsible for development of the gut immune system in a rodent model. Microbes Infect 2, 1343-1351.

13. Eberl G \& Lochner M (2009) The development of intestinal lymphoid tissues at the interface of self and microbiota. Mucosal Immunol 2, 478-485.

14. Handelsman J, Rondon MR, Brady SF, et al. (1998) Molecular biological access to the chemistry of unknown soil microbes: a new frontier for natural products. Chem Biol 5, R245-R249. 
15. Frank DN \& Pace NR (2008) Gastrointestinal microbiology enters the metagenomics era. Curr Opin Gastroenterol 24, $4-10$.

16. Turnbaugh PJ, Ley RE, Hamady M, et al. (2007) The human microbiome project. Nature 449, 804-810.

17. Eckburg PB, Bik EM, Bernstein CN, et al. (2005) Diversity of the human intestinal microbial flora. Science 308, 1635-1638.

18. Qin J, Li R, Raes J, et al. (2010) A human gut microbial gene catalogue established by metagenomic sequencing. Nature 464, 59-65.

19. Arumugam M, Raes J, Pelletier E, et al. (2011) Enterotypes of the human gut microbiome. Nature 473, 174-180.

20. Huse SM, Ye Y, Zhou Y, et al. (2012) A core human microbiome as viewed through 16S rRNA sequence clusters. PLoS One 7, e34242.

21. Gatt M, Reddy BS \& MacFie J (2007) Review article: bacterial translocation in the critically ill - evidence and methods of prevention. Aliment Pharmacol Ther 25, 741-757.

22. Ley RE, Turnbaugh PJ, Klein S, et al. (2006) Microbial ecology: human gut microbes associated with obesity. Nature 444, 1022-1023.

23. Blaut M \& Klaus S (2012) Intestinal microbiota and obesity. Handb Exp Pharmacol 209, 251-273.

24. Guarner F (2008) What is the role of the enteric commensal flora in IBD? Inflamm Bowel Dis 14, S83-S84.

25. Sokol H, Pigneur B, Watterlot L, et al. (2008) Faecalibacterium prausnitzii is an anti-inflammatory commensal bacterium identified by gut microbiota analysis of Crohn disease patients. Proc Natl Acad Sci U S A 105, 16731-16736.

26. Frank DN, St Amand AL, Feldman RA, et al. (2007) Molecular-phylogenetic characterization of microbial community imbalances in human inflammatory bowel diseases. Proc Natl Acad Sci U S A 104, 13780-13785.
27. Van Nimwegen FA, Penders J, Stobberingh EE, et al. (2011) Mode and place of delivery, gastrointestinal microbiota, and their influence on asthma and atopy. Allergy Clin Immunol 128, 948-955.e1-3.

28. Penders J, Thijs C, van den Brandt PA, et al. (2007) Gut microbiota composition and development of atopic manifestations in infancy: the KOALA Birth Cohort Study. Gut 56, 661-667.

29. Duboc H (2012) Increase in fecal primary bile acids and dysbiosis in patients with diarrhea-predominant irritable bowel syndrome. Neurogastroenterol Motil 24, 513-520.

30. Song Y, Liu C \& Finegold SM (2004) Real-time PCR quantitation of clostridia in feces of autistic children. Appl Environ Microbiol 70, 6459-6465.

31. Finegold SM, Dowd SE, Gontcharova V, et al. (2010) Pyrosequencing study of fecal microflora of autistic and control children. Anaerobe 16, 444-453.

32. WGO Practice Guideline - Probiotics and Prebiotics. http://www.worldgastroenterology.org/probiotics-prebiotics. html

33. Roberfroid M, Gibson GR, Hoyles L, et al. (2010) Prebiotic effects: metabolic and health benefits. Br J Nutr 104, Suppl. 2, S1-S63.

34. Coppa GV, Bruni S, Morelli L, et al. (2004) The first prebiotics in humans: human milk oligosaccharides. J Clin Gastroenterol 38, Suppl. 6, S80-S83.

35. Grüber C, van Stuijvenberg M \& Mosca F (2010) Reduced occurrence of early atopic dermatitis because of immunoactive prebiotics among low-atopy-risk infants. J Allergy Clin Immunol 126, 791-797.

36. Landy J (2011) Review article: faecal transplantation therapy for gastrointestinal disease. Aliment Pharmacol Ther 34, 409-415. 